\title{
Safety and feasibility of single-port laparoscopic multivisceral resection for locally advanced left colon cancer
}

\author{
MITSUYOSHI TEI, MASAHISA OTSUKA, YOZO SUZUKI, \\ KENTARO KISHI, MASAHIRO TANEMURA and HIROKI AKAMATSU \\ Department of Surgery, Osaka Police Hospital, Osaka 543-0035, Japan
}

Received November 22, 2017; Accepted March 16, 2018

DOI: $10.3892 /$ ol.2018.8582

\begin{abstract}
The aim of the present study was to evaluate the safety and feasibility of single-port laparoscopic multivisceral resection (S-MVR) for locally advanced left colon cancer. S-MVR is a challenging technique and to the best of our knowledge this is the first report of S-MVR for left colon cancer invading or adhering to neighboring organs. A retrospective review was conducted of patients who underwent laparoscopic multivisceral resection for locally advanced left colon cancer invading or adhering to neighboring organs from January 2008 to December 2014. Short-term and long-term outcomes were analyzed between groups of patients who underwent S-MVR and multi-port laparoscopic multivisceral resection (M-MVR) retrospectively. A total of 14 patients underwent S-MVR and 15 patients underwent M-MVR. There were no significant differences between groups in terms of operative factors and postoperative complications. The length of hospital stay was significantly shorter in the S-MVR group compared with the M-MVR group $(\mathrm{P}=0.048)$. Three-year overall survival was $61.9 \%$ in the S-MVR group $(n=14)$. In patients with stage II $(\mathrm{P}=0.600)$ and III $(\mathrm{P}=0714)$ disease the three-year overall and disease-free survival was 81.8 and $58.3 \%$ in the S-MVR group and 80.0 and $70 \%$ in the M-MVR groups over a median follow-up of 34 months. In conclusion, S-MVR for locally advanced left colon cancer is safe and feasible in selected patients.
\end{abstract}

\section{Introduction}

The results of several prospective randomized trials have shown the benefits of laparoscopic surgery for localized colorectal cancer in terms of postoperative outcomes, including reduction of pain, earlier intestinal peristaltic recovery, shortening of postoperative stay, cosmetic advantages, and equivalent

Correspondence to: Dr Mitsuyoshi Tei, Department of Surgery, Osaka Police Hospital, 10-31 Kitayama-cho, Tennoji-ku, Osaka 543-0035, Japan

E-mail: mtei@live.jp

Key words: single-incision laparoscopic surgery, single-port surgery, multivisceral resection, left colon cancer effectiveness on long-term cancer outcomes (1-4). However, locally advanced colon cancers sometimes invade or adhere to neighboring organs or structures, and en bloc multivisceral resection is necessary. According to the European Association of Endoscopic Surgery (EAES) consensus, potentially curative resection of colon cancer suspected of invading the abdominal wall or adjacent structures should be undertaken by open surgery (5). In the Society of American Gastrointestinal and Endoscopic Surgeons (SAGES) guidelines, as open approach is suggested if a laparoscopic en bloc resection cannot be performed adequately for locally advanced adherent colon tumors (6). Because of increased the experience with laparoscopic approach for locally advanced colon cancer, the opportunities for adopting laparoscopic surgery for advanced colon cancer invading or adhering to neighboring organs or structures are considered to be increasing. Several studies have demonstrated that laparoscopic multivisceral resection for advanced colorectal cancer was safe and feasible in selected patients (7-9).

The first single-port surgery (SPS) procedure was described for right colectomy in 2008 (10). Compared with conventional multi-port surgery, the benefits of SPS include better cosmetic outcomes, less postoperative pain (11-13), faster postoperative recovery (14), and earlier discharge from the hospital $(13,14)$. SPS for colorectal cancer was shown to be safe, feasible $(11,12,15)$, and able to provide perioperative and short-term oncological outcomes equal to those of multi-port surgery $(16,17)$. However, cases of locally advanced colon cancer invading or adhering to neighboring organs were excluded in these studies (11-17), and there is no study of SPS for locally advanced left colon cancer invading or adhering to neighboring organs. Therefore, in this study, we aimed to evaluate the safety and feasibility of single-port laparoscopic multivisceral resection (S-MVR) for locally advanced left colon cancer invading or adhering to neighboring organs, based on a retrospective comparison with multi-port laparoscopic multivisceral resection (M-MVR).

\section{Patients and methods}

Patient profiles. Multivisceral resection was defined as en bloc resection of any organ or structure to which the primary tumor was adherent. The preoperative diagnosis of cancer was established with a barium enema, a colonoscopy, and 
computed tomography for evaluation of local disease and distant metastases. Locally advanced colon cancer was diagnosed with preoperative computed tomography when the tumor and surrounding structures were unclear. In cases with unclear boundaries, we judged whether the tumor invaded adjacent organs or structures during the intraoperative period. Patients who had a diagnosis of Stage IV disease who underwent macroscopic complete resection of the primary tumor were included in this study, but those who underwent palliative resection of the primary tumor were excluded.

In our department, the first SPS for benign disease was carried out in May 2009. Before January 2010, SPS was performed only for benign disease and early colon cancer only, but the indication was expanded later to include advanced colorectal cancer. SPS for locally advanced colon cancer invading or adhering to neighboring organs has been performed since January 2011, and since then, it has been considered the primary treatment for colon cancer in those eligible for conventional multi-port surgery.

Between January 2008 and December 2014, a total of 38 patients underwent multivisceral resection for locally advanced left colon cancer invading or adhering to neighboring organs at our department. Among these patients, 9 patients underwent open surgery because of an emergency operation $(n=3)$, a tumor associated abscess $(n=2)$. In addition, we performed open surgery when urinary diversion and total cystectomy were needed $(n=4)$. In total, 29 consecutive patients who underwent S-MVR or M-MVR for locally advanced left colon cancer with adjacent organs or structures in this study. Patients received written information describing the differences between S-MVR and M-MVR. All of the patients agreed to receive S-MVR and provided written informed consent. The study was approved by the Ethics Committee of Osaka Police Hospital.

Data collection. For each patient, the following variables were collected from our prospectively maintained database; age, sex, body mass index (BMI), American Society of Anesthesiology Score (ASA score), previous abdominal surgery, tumor location, tumor size, operative procedure, stoma construction, operative time, blood loss, conversion rate, morbidity, mortality, length of hospital stay, and pathological findings. Postoperative complications were classified according to the Dindo et al Classification (18). Operative mortality was defined as death on the same admission or within 30 days of surgery. All patients were followed for at least 30 days after surgery.

Surgical procedure. SPS was performed, as described previously (19). A flexible laparoscope $10 \mathrm{~mm}$ in diameter and only standard straight laparoscopic instruments were used. Every procedure was performed with an Ultracision harmonic scalpel regardless of whether it was single or multi-port surgery. High ligation of feeding vessels and en bloc resection of the primary tumor with a safe margin around the adjacent organs or structures were performed. For left colectomy, an extracorporeal functional end-to-end anastomosis was then created. For sigmoidectomy and high anterior resection, the sigmoid colon or upper rectum was divided laparoscopically with staplers. An end-to-end anastomosis was created with the trans-anal insertion of a circular stapler.
Statistical analysis. Continuous data are expressed as median (range), unless specifically noted. Preoperative factors, operative factors, and pathological outcomes were analyzed retrospectively. Statistical analyses were performed with the use of JMP 11.0 software (SAS Institute Inc., Cary, NC, USA). All group data are reported as median values. Differences in age, BMI, tumor size, operative time, blood loss, length of hospital stay, and number of harvested lymph nodes were analyzed with Mann-Whitney's U test. Other factors were analyzed with Fischer's exact probability test. Disease-free survival and overall survival rate were determined using Kaplan-Meier analysis with a log-rank test. All analyses were performed on an intention-to-treat basis: patients who had S-MVR converted to M-MVR were included in the S-MVR group. Patients who had M-MVR converted to open surgery were included in the M-MVR group. $\mathrm{P}<0.05$ was considered to indicate a statistically significant difference.

\section{Results}

Table I lists the profiles of the patients analyzed by group. There were no significant differences between the S-MVR and M-MVR groups in terms of age, gender, BMI, ASA score, previous abdominal surgery, tumor location and tumor size. None of the patients received neoadjuvant chemotherapy or chemoradiotherapy in this study.

Table II summarizes the operative factors. The conversion rate in the S-MVR group was $14.3 \%$ (2/14 patients). None of the patients who underwent S-MVR was converted to open surgery. The reason for conversion to multi-port surgery was strong inflammatory adhesion to the small intestine in one patients and bladder invasion in one patient. In the M-MVR group, 5 patients were converted to open surgery because of tumor invasion of the small intestine in three, invasion of the right ureter and the abdominal wall in one, and invasion of the uterus in one. There were no significant differences between groups in terms of operative procedure, stoma construction rate (14.3\% in the S-MVR group vs. 26.7\% in the M-MVR group, $\mathrm{P}=0.651$ ), operative time (222 min in the $\mathrm{S}-\mathrm{MVR}$ group vs. $255 \mathrm{~min}$ in the M-MVR group, $\mathrm{P}=0.163)$, blood loss (60 $\mathrm{ml}$ in the S-MVR group vs. $220 \mathrm{ml}$ in the M-MVR group, $\mathrm{P}=0.295$ ), and the median number of harvested lymph nodes (30 in the S-MVR group vs. 25 in the M-MVR group, $\mathrm{P}=0.328$ ).

Table III lists the adjacent organs or structures resected en bloc. The most commonly affected organs were the small intestine (28.6\% in the S-MVR group and $46.7 \%$ in the M-MVR group). The number of resected organs or structures was similar between the two groups $(\mathrm{P}=1.000)$.

Table IV summarizes the postoperative complications in each group. The overall complication rate was $28.6 \%$ in the S-MVR group. There were no significant differences between groups in terms of postoperative complication. Length of hospital stay was significantly shorter with S-MVR than with M-MVR (11 vs. 18 days, $\mathrm{P}=0.048$ ). Reoperation was performed in one patient in the M-MVR group because of anastomotic leakage. Perioperative death was not recorded in both groups.

Table V summarizes the pathological findings for each group. The R0 resection rate of the primary tumor was similar between groups $(100 \%$ in the S-MVR group and $93.3 \%$ in the M-MVR group). The rate of pathological 
Table I. Patient profiles.

\begin{tabular}{|c|c|c|c|}
\hline Characteristic & S-MVR $(n=14)$ & M-MVR (n=15) & P-value \\
\hline Median age, years (range) & $71(36-89)$ & $69(53-91)$ & 0.878 \\
\hline \multicolumn{4}{|l|}{ Sex, n } \\
\hline Male/female & $6 / 8$ & $10 / 5$ & 0.272 \\
\hline Median BMI, kg/m² (range) & $21.5(14.1-32.9)$ & $19.4(12.5-30.5)$ & 0.176 \\
\hline ASA score, $n$ & & & 0.814 \\
\hline 1 or $2 / \geq 3$ & $12 / 2$ & $12 / 3$ & \\
\hline Previous abdominal surgery, $\mathrm{n}$ & 4 & 3 & 0.682 \\
\hline Tumor location, $\mathrm{n}$ & & & 0.330 \\
\hline Descending/sigmoid & $3 / 11$ & $1 / 14$ & \\
\hline Median tumor size, mm (range) & $60(25-110)$ & $70(40-110)$ & 0.333 \\
\hline
\end{tabular}

ASA score, American Society of Anesthesiology Score; BMI, body mass index; S-MVR, single-port laparoscopic multivisceral resection; M-MVR, multi-port laparoscopic multivisceral resection.

Table II. Comparison of operative factors.

\begin{tabular}{|c|c|c|c|}
\hline Factors & S-MVR (n=14) & M-MVR (n=15) & P-value \\
\hline Operative procedure, $\mathrm{n}$ & & & 0.591 \\
\hline Left hemicolectomy & 3 & 1 & \\
\hline Sigmoidectomy & 8 & 9 & \\
\hline High anterior resection & 3 & 5 & \\
\hline Stoma construction, $\mathrm{n}$ & 2 & 4 & 0.651 \\
\hline Median operative time, min (range) & $222(129-323)$ & $255(174-395)$ & 0.163 \\
\hline Median blood loss, ml (range) & $60(5-600)$ & $220(5-2670)$ & 0.295 \\
\hline Median number of harvested lymph nodes, (range) & $30(17-58)$ & $25(10-72)$ & 0.328 \\
\hline Conversion to MPS, $\mathrm{n}$ & 2 & - & - \\
\hline Conversion to open surgery, $\mathrm{n}$ & 0 & 5 & - \\
\hline
\end{tabular}

S-MVR, single-port laparoscopic multivisceral resection; M-MVR, multi-port laparoscopic multivisceral resection.

T4b disease was $35.7 \%$ in the S-MVR group and $33.3 \%$ in the M-MVR group $(\mathrm{P}=1.000)$. There were no significant differences between groups in terms of the grade of differentiation, pathological lymph node metastasis, and TNM classification.

Table VIlists the detail of cancer recurrence in both groups. 4 patients in the S-MVR group and 3 patients in the M-MVR group had distant metastases. 4 patients had a diagnosis of pathological T4b. 5 of 7 patients had received postoperative adjuvant chemotherapy. None of 7 patients had received a secondary intervention. There was no significant difference between groups in the 3 -year overall survival rate $(61.9 \%$ in the S-MVR group vs. $58.7 \%$ in the M-MVR group, $\mathrm{P}=0.777$ ) over a median follow-up period of 30.7 (2.0-63.9) months (Fig. 1). In pathologic Stage II or III patients, the 3 -year overall survival rate was $81.8 \%$ in the S-MVR group and $80.0 \%$ in the M-MVR group ( $\mathrm{P}=0.600)$ ( Fig. 2). The 3 year disease-free survival rate was $58.3 \%$ and in the S-MVR group and $70.0 \%$ in the M-MVR group $(\mathrm{P}=0.714)$ over a median follow-up period of 34.0 (2.0-63.9) months (Fig. 3).

\section{Discussion}

Previously, we reported that SPS was safe, feasible and could provide perioperative and short-term oncological outcomes equal to those of multi-port surgery $(15,16)$. S-MVR is a somewhat challenging technique, and we consider that maintaining the regional oncologic outcome is the most important factor for surgical treatment. In our series, the R0 resection rate of the primary tumor was $100 \%$ in the S-MVR group, and similar with the M-MVR group. No gross or microscopic positive resection margins were observed in the S-MVR group. The median number of harvested lymph nodes was 30 in the S-MVR group, and did not differ compared with the M-MVR group, which exceeded that of other reports (7-9). To the best of our knowledge, this is the first study to report the 
Table III. Organs or structures resected at multivisceral resection.

\begin{tabular}{lccc}
\hline Organ or structure & S-MVR (n=14) & M-MVR (n=15) & P-value \\
\hline Small intestine & 4 & 2 & 0.450 \\
Abdominal wall & 3 & 2 & 0.651 \\
Urinary bladder & 3 & 1 & 0.651 \\
Ovary & 3 & 1 & 0.330 \\
Appendix & 1 & 0 & 0.776 \\
Seminal vesicle & 1 & 2 & 0.483 \\
Uterus & 1 & 1 & 0.527 \\
Ureter & 0 & 18 & 0.517 \\
Total & 17 & $12 / 3$ \\
Number of resected organs or structure & & $11 / 3$ & 0.709 \\
$1 / 2 /$ & & & \\
\hline
\end{tabular}

S-MVR, single-port laparoscopic multivisceral resection; M-MVR, multi-port laparoscopic multivisceral resection.

Table IV. Postoperative complications.

\begin{tabular}{lccc}
\hline Complication & S-MVR (n=14) & M-MVR (n=15) & 0 \\
\hline Bleeding & 1 & 1 & 0.483 \\
Anastomotic leakage & 0 & 3 & 0517 \\
Wound infection & 2 & 1 & 0.814 \\
Abdominal abscess & 2 & 1 & 0.598 \\
Pneumonia & 0 & 2 & 0.517 \\
Urinary tract infection & 1 & 2 & 0.527 \\
Bowel obstruction & 0 & 0 & 0.483 \\
Colitis & 1 & 1 & 0.483 \\
Reoperation & 0 & 0 & 0.517 \\
Perioperative death & 0 & 7 & - \\
Overall complications & 4 & $18(8-90)$ \\
Length of hospital stay, days (range) & $11(7-97)$ & 0.450 \\
\hline
\end{tabular}

S-MVR, single-port laparoscopic multivisceral resection; M-MVR, multi-port laparoscopic multivisceral resection.

clinical outcomes of S-MVR for locally advanced left colon cancer.

In this study, we showed that S-MVR and M-MVR do not differ significantly in terms of blood loss, operative time and number of harvested lymph nodes. The operative time in the present study was comparable with that of other reports (7-9). S-MVR was performed successfully in $78.6 \%$ of patients. Some studies of SPS have reported that the conversion rate to MPS or open surgery ranged from 1.4 to $10.9 \%(11,12,14)$. Previous reports have demonstrated the safety and feasibility of SPS in colorectal cancer, but their inclusion criteria yielded highly selected patients (with early cancer or small tumors) (11,12). The EAES consensus and SAGES guidelines do not necessary recommend laparoscopic en bloc resection for locally advanced adherent colon cancers $(5,6)$. However, our results with S-MVR for advanced primary colon cancer had high reliability in terms of oncological clearance, blood loss, operative time and a successful completion rate regarding the regional oncologic aspect.

In this study, the rate of pathological tumor invasion of adjacent organs or structures (pT4b) was $35.7 \%$ in the SPS group, and did not differ compared with the MPS group $(\mathrm{P}=1.000)$. A previous review reported that pathological true infiltration is present in $54.1 \%$ of patients who underwent multivisceral resection for colorectal cancer, whereas peritumoral adhesion can be caused by an inflammatory reaction in the remaining patients (20). Because it is very difficult to judge whether the adhesion is due to true tumor invasion or an inflammatory reaction intraoperatively, it is important to resect the primary colon cancer en bloc with adjacent organ, preventing exposure and dissemination of cancer cells.

We showed that the overall postoperative complication rate in the SPS group was $28.6 \%$, and did not differ compared with the MPS group $(\mathrm{P}=0.450)$. None of the patients in the 
Table V. Pathological outcomes.

\begin{tabular}{|c|c|c|c|}
\hline Outcome & S-MVR $(n=14)$ & M-MVR (n=15) & P-value \\
\hline Residual tumor status of the primary tumor & & & 0.517 \\
\hline R0/R1 & $14 / 0$ & $13 / 1$ & \\
\hline Grade of differentiation, $n$ & & & 0.814 \\
\hline Well or moderate/poor or mucinous & $12 / 2$ & $11 / 3$ & \\
\hline Depth of tumor invasion, $\mathrm{n}$ & & & 0.992 \\
\hline $\mathrm{T} 3$ & 2 & 1 & \\
\hline $\mathrm{T} 4 \mathrm{a}$ & 7 & 9 & \\
\hline $\mathrm{T} 4 \mathrm{~b}$ & 5 & 5 & \\
\hline Lymph node metastasis, $\mathrm{n}$ & & & 0.449 \\
\hline No & 5 & 9 & \\
\hline $\mathrm{N} 1$ & 4 & 3 & \\
\hline $\mathrm{N} 2$ & 5 & 3 & \\
\hline TNM classification, $\mathrm{n}$ & & & 0.461 \\
\hline II & 4 & 6 & \\
\hline III & 7 & 4 & \\
\hline IV & 3 & 5 & \\
\hline
\end{tabular}

S-MVR, single-port laparoscopic multivisceral resection; M-MVR, multi-port laparoscopic multivisceral resection.

Table VI. Cancer recurrence.

\begin{tabular}{|c|c|c|c|c|c|c|c|}
\hline Sex & Age & $\begin{array}{c}\text { Operation } \\
\text { type }\end{array}$ & $\begin{array}{l}\text { Tumor } \\
\text { depth }\end{array}$ & Stage & $\begin{array}{l}\text { Timing of } \\
\text { recurrence }\end{array}$ & Recurrence pattern & Prognosis \\
\hline M & 43 & S-MVR & $\mathrm{T} 4 \mathrm{~b}$ & III & $6 \mathrm{~m}$ & Liver, peritoneum & 3 y 6 m deceased \\
\hline M & 51 & S-MVR & $\mathrm{T} 4 \mathrm{~b}$ & III & $6 \mathrm{~m}$ & Liver, lung, peritoneum, para-aortic lymph node & $7 \mathrm{~m}$ deceased \\
\hline $\mathrm{F}$ & 85 & S-MVR & $\mathrm{T} 4 \mathrm{a}$ & III & $6 \mathrm{~m}$ & Lung & 2 y $10 \mathrm{~m}$ alive \\
\hline $\mathrm{F}$ & 76 & S-MVR & $\mathrm{T} 4 \mathrm{a}$ & III & $1 \mathrm{y} 4 \mathrm{~m}$ & Lung, & 3 y $2 \mathrm{~m}$ alive \\
\hline $\mathrm{F}$ & 83 & M-MVR & $\mathrm{T} 4 \mathrm{~b}$ & III & $7 \mathrm{~m}$ & Peritoneum, para-aortic lymph node & 3 y 6 m deceased \\
\hline M & 81 & M-MVR & $\mathrm{T} 4 \mathrm{a}$ & III & $7 \mathrm{~m}$ & Virchow lymph node & 2 y $10 \mathrm{~m}$ deceased \\
\hline M & 63 & M-MVR & $\mathrm{T} 4 \mathrm{~b}$ & III & $3 \mathrm{~m}$ & Liver, lung, peritoneum & $6 \mathrm{~m}$ deceased \\
\hline
\end{tabular}

S-MVR, single-port laparoscopic multivisceral resection; M-MVR, multi-port laparoscopic multivisceral resection; M, male; F, female; $\mathrm{y}$, years; $\mathrm{m}$, months.

SPS group experienced perioperative death. Previous studies of laparoscopic multivisceral resection have reported that the morbidity ranged from 21 to $33 \%$ (7-9), and our series was comparable with those of the previous studies. The incidence of complications in the MPS group in the present study was higher compared with that of other reports (7-9). Even minor complications that might have little or no influence on postoperative hospital stay or mortality were counted. Nevertheless, we understand that the rate of complications is within an acceptable range. Regarding the length of hospital stay, recent studies in Western countries indicate a median or mean postoperative hospital stay of 5 to 10 days, whereas the median hospital stay was 11 days in the SPS group and 18 days in the MPS group in this study. The reason of longer hospital stay is that the Japanese health insurance system, which maintains low medical costs, has influenced this result.
Our perioperative outcomes of single-port multivisceral resection might be explained by two factors. First, we selected the patients carefully according to whether S-MVR was possible. In this study, none of patients underwent total cystectomy and urinary diversion. Laparoscopic total cystectomy and intracorporeal urinary diversion are technically challenging under robotic assistance, and many surgeons are still adopting the hybrid approach when performing the cystectomy using robotic assistance and completing the urinary diversions extracorporeally (21). We consider that open surgery should be chosen if total cyctectomy and urinary diversion are needed. Second, we have experience in carrying out a large number of single-port laparoscopic colorectal surgeries. Therefore, the frequency of single-port multivisceral resections is increasing gradually at our department. It is very important 


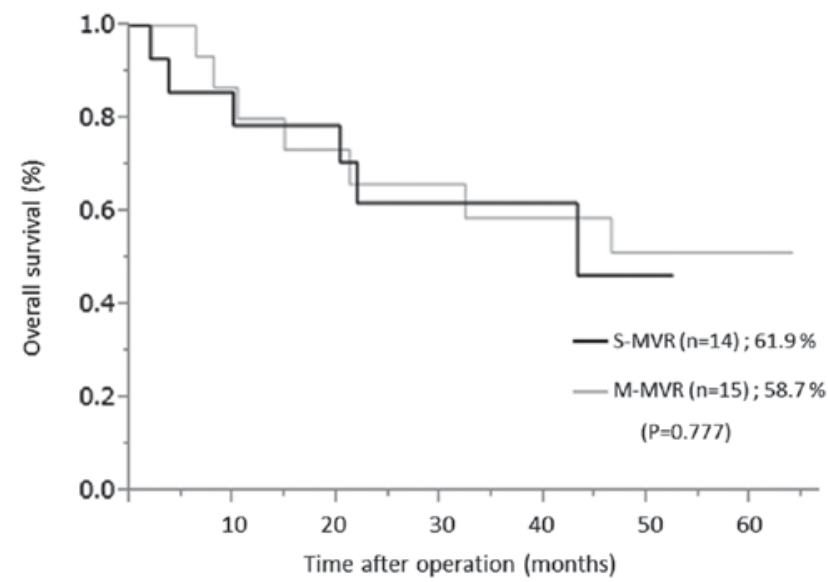

Figure 1. Kaplan-Meier analysis of overall survival at 3 years for all patients between the S-MVR and M-MVR group ( $\mathrm{P}=0.777)$. S-MVR, single-port laparoscopic multivisceral resection; M-MVR, multi-port laparoscopic multivisceral resection.

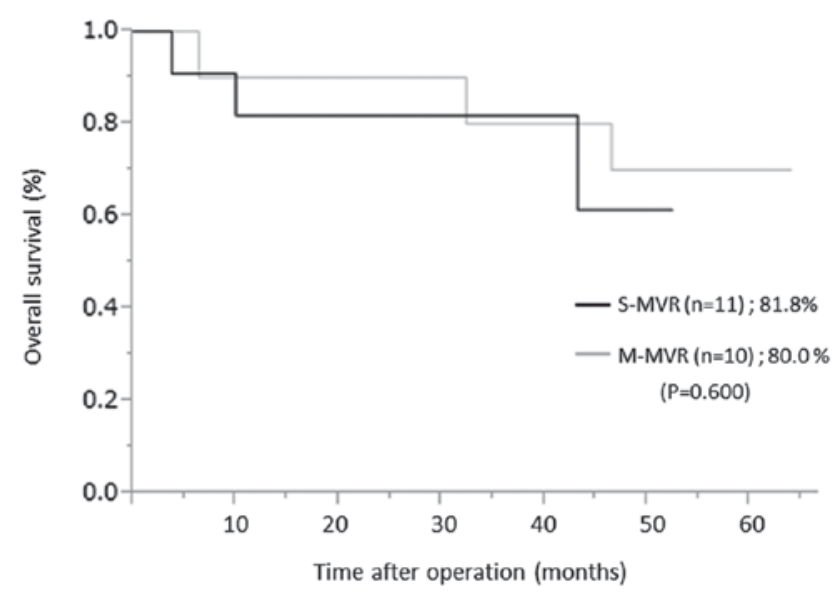

Figure 2. Kaplan-Meier analysis of overall survival at 3 years for pathological Stage II or III patients between the S-MVR and M-MVR group $(\mathrm{P}=0.600)$. S-MVR, single-port laparoscopic multivisceral resection; M-MVR, multi-port laparoscopic multivisceral resection.

to select optimal cases and perform single-port laparoscopic colorectal surgeries adequately for successful single-port multivisceral resection.

We showed that the pathological R0 resection rate was $100 \%$ in the SPS group. High vascular ligation and a non-touch technique were maintained in this series. None of patients in the SPS group had regional lymph nodes recurrence. However, 4 patients had distant metastases in the SPS group. Cukier et al reported that 3 year overall survival and disease-free survival rate was 85.9 and $73.7 \%$, respectively (22). López-Cano et al reported that 5 -year overall disease-free survival rate was $48 \%$ (23). Luna-Perez et al reported that 5-year overall survival rate was $45 \%$ over a median follow-up period of 36.8 months (24). Our oncological outcome was comparable with those of the previous studies regardless of operative approach.

The main limitations of this study are that it was carried out at a single institution and that it was very small and retrospective in nature. Therefore, it contains selection bias and an inability to match the backgrounds of patients between groups. In addition,

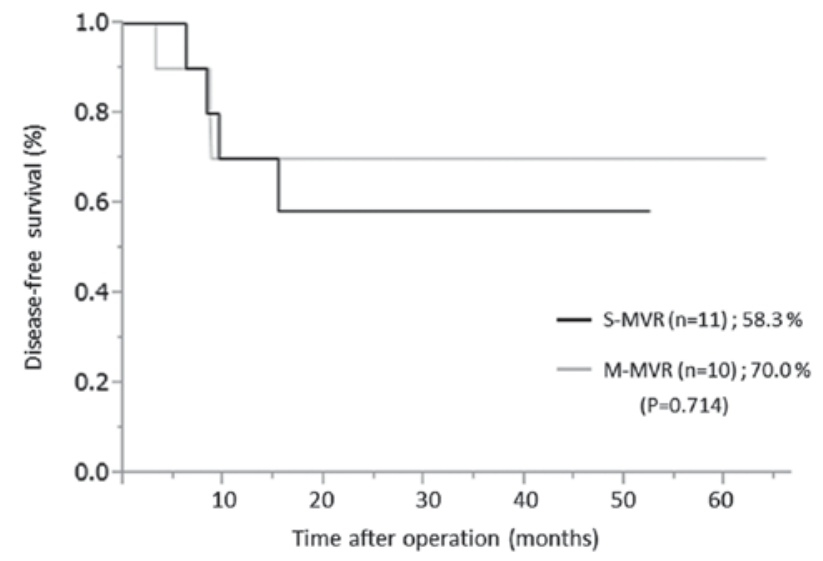

Figure 3. Kaplan-Meier analysis of disease-free survival at 3 years for pathological Stage II or III patients between the S-MVR and M-MVR group $(\mathrm{P}=0.714)$. S-MVR, single-port laparoscopic multivisceral resection; M-MVR, multi-port laparoscopic multivisceral resection.

our total patient population may be typical for Japan and not be applicable to the average European or United States population. However, we believe that when patients are selected appropriately, the single-port multivisceral resection has no adverse effects on perioperative and short-term oncological outcomes. More studies are needed to validate our results and prove the true value of the single-port multivisceral resection.

To the best of our knowledge his is the first report of S-MVR for left colon cancer invading or adhering to neighboring organs. S-MVR in locally advanced left colon cancer is safe and feasible in appropriately selected patients.

\section{Acknowledgements}

Not applicable.

\section{Funding}

No funding was received.

\section{Availability of data and materials}

All data generated or analyzed during this study are included in this published article.

\section{Authors' contributions}

MTe drafted the paper and analyzed and interpreted data. MO, YS, KK and MTa made substantial contributions to the acquisition of the data. HA made substantial contributions to the concept and design of the study.

\section{Ethics approval and consent to participate}

The study was approved by the Ethics Committee of Osaka Police Hospital.

\section{Consent for publication}

Not applicable. 


\section{Competing interests}

The authors declare that they have no competing interests.

\section{References}

1. Lacy AM, García-Valdecasas JC, Delgado S, Castells A, Taurá P, Piqué JM and Visa J: Laparoscopy-assisted colectomy versus open colectomy for treatment of non-metastatic colon cancer: A randomised trial. Lancet 359: 2224-2229, 2002.

2. Milsom JW, Böhm B, Hammerhofer KA, Fazio V, Steiger E and Elson P: A prospective, randomized trial comparing laparoscopic versus conventional techniques in colorectal cancer surgery: A preliminary report. J Am Coll Surg 187: 46-54; Discussion 54-55, 1998.

3. Leung KL, Kwok SP, Lam SC, Lee JF, Yiu RY, Ng SS, Lai PB and Lau WY: Laparoscopic resection of rectosigmoid carcinoma: Prospective randomised trial. Lancet 363: 1187-1192, 2004.

4. Clinical Outcomes of Surgical Therapy Study Group, Nelson H, Sargent DJ, Wieand HS, Fleshman J, Anvari M, Stryker SJ, Beart RW Jr, Hellinger M, Flanagan R Jr, et al: A comparison of laparoscopically assisted and open colectomy for colon cancer. N Engl J Med 350: 2050-2059, 2004.

5. Veldkamp R, Gholghesaei M, Bonjer HJ, Meijer DW, Buunen M, Jeekel J, Anderberg B, Cuesta MA, Cuschierl A, Fingerhut A, et al: Laparoscopic resection of colon cancer: Consensus of the European Association of Endoscopic Surgery (EAES). Surg Endosc 18: 1163-1185, 2004

6. Zerey M, Hawver LM, Awad Z, Stefanidis D, Richardson W and Fanelli RD; Members of the SAGES Guidelines Committee: SAGES evidence-based guidelines for the laparoscopic resection of curable colon and rectal cancer. Surg Endosc 27: 1-10, 2013.

7. Kim KY, Hwang DW, Park YK and Lee HS: A single surgeon's experience with 54 consecutive cases of multivisceral resection for locally advanced primary colorectal cancer: Can the laparoscopic approach be performed safely? Surg Endosc 26: 493-500, 2012.

8. Nagasue Y, Akiyoshi T, Ueno M, Fukunaga Y, Nagayama S, Fujimoto Y, Konishi T, Nagasaki T, Nagata J, Mukai T, et al: Laparoscopic versus open multivisceral resection for primary colorectal cancer: Comparison of perioperative outcomes J Gastrointest Surg 17: 1299-1305, 2013.

9. Bretagnol F, Dedieu A, Zappa M, Guedj N, Ferron M and Panis Y: T4 colorectal cancer: Is laparoscopic resection contraindicated? Colorectal Dis 13: 138-143, 2011.

10. Remzi FH, Kirat HT, Kaouk JH and Geisler DP: Single-port laparoscopy in colorectal surgery. Colorectal Dis 10: 823-826, 2008

11. Takemasa I, Uemura M, Nishimura J, Mizushima $T$, Yamamoto H, Ikeda M, Sekimoto M, Doki Y and Mori M: Feasibility of single-site laparoscopic colectomy with complete mesocolic excision for colon cancer: A prospective case-control comparison. Surg Endosc 28: 1110-1118, 2014.
12. Champagne BJ, Papaconstantinou HT, Parmar SS, Nagle DA, Young-Fadok TM, Lee EC and Delaney CP: Single-incision versus standard multiport laparoscopic colectomy: A multicenter, case-controlled comparison. Ann Surg 255: 66-69, 2012.

13. Poon JT, Cheung CW, Fan JK, Lo OS and Law WL: Single-incision versus conventional laparoscopic colectomy for colonic neoplasm: A randomized, controlled trial. Surg Endosc 26: 2729-2734, 2012.

14. Kim SJ, Ryu GO, Choi BJ, Kim JG, Lee KJ, Lee SC and Oh ST: The short-term outcomes of conventional and single-port laparoscopic surgery for colorectal cancer. Ann Surg 254: 933-940, 2011.

15. Tei $\mathbf{M}$, Wakasugi $\mathrm{M}$ and Akamatsu $\mathrm{H}$ : Comparison of short-term surgical results of single-port and multi-port laparoscopic rectal resection for rectal cancer. Am J Surg 210: 309-314, 2015.

16. Tei M, Wakasugi $\mathrm{M}$ and Akamatsu $\mathrm{H}$ : Comparison of the perioperative and short-term oncological outcome after single- or multiport surgery for colorectal cancer. Colorectal Dis 17: O141-O147, 2015.

17. Yun JA, Yun SH, Park YA, Huh JW, Cho YB, Kim HC and Lee WY: Oncologic outcomes of single-incision laparoscopic surgery compared with conventional laparoscopy for colon cancer. Ann Surg 263: 973-978, 2016.

18. Dindo D, Demartines N and Clavien PA: Classification of surgical complications: A new proposal with evaluation in a cohort of 6336 patients and results of a survey. Ann Surg 240: 205-213, 2004

19. Tei M, Wakasugi M, Omori T, Ueshima S, Tori $M$ and Akamatsu H: Single-port laparoscopic colectomy is safe and feasible in patients with previous abdominal surgery. Am J Surg 209: 1007-1012, 2015.

20. Mohan HM, Evans MD, Larkin JO, Beynon J and Winter DC: Multivisceral resection in colorectal cancer: A systematic review. Ann Surg Oncol 20: 2929-2936, 2013.

21. Sim A, Balbay MD, Todenhöfer T, Aufderklamm S, Halalsheh O, Mischinger J, Böttge J, Rausch S, Bier S, Stenzl A, et al: Robot-assisted radical cystectomy and intracorporeal urinary diversion-safe and reproducible? Cent European J Urol 68: 18-23, 2015.

22. Cukier M, Smith AJ, Milot L, Chu W, Chung H, Fenech D, Herschorn S, Ko Y, Rowsell C, Soliman H, et al: Neoadjuvant chemoradiotherapy and multivisceral resection for primary locally advanced adherent colon cancer: A single institution experience. Eur J Surg Oncol 38: 677-682, 2012.

23. López-Cano M, Mañas MJ, Hermosilla E and Espín E: Multivisceral resection for colon cancer: Analysis of prognostic factors. Dig Surg 27: 238-245, 2010.

24. Luna-Pérez P, Rodríguez-Ramírez SE, De la Barrera MG, Zeferino $\mathrm{M}$ and Labastida S: Multivisceral resection for colon cancer. J Surg Oncol 80: 100-104, 2002. 